\title{
Mathematical Model for Calculation of Process of Chemisorption Adsorption of Carbon Dioxide
}

\author{
Guzel R. Kamaletdinova \\ Tambov State Technical University \\ Tambov, Russia \\ kamaletdinova.guzel@gmail.com
}

\author{
Sergey A. Skvortsov \\ Tambov State Technical University \\ Tambov, Russia \\ dfoxd@rambler.ru
}

\author{
Maxim P. Onevsky \\ Tambov State Technical University \\ Tambov, Russia \\ maxim.onevsky@gmail.com
}

\author{
Alexander A. Tret'yakov \\ Tambov State Technical University \\ Tambov, Russia \\ tsasha74@mail.ru
}

\author{
Alexander N. Pchelintsev \\ Tambov State Technical University \\ Tambov, Russia \\ pchelintsev.an@yandex.ru
}

\begin{abstract}
The system of assumptions of the mathematical description of the process of chemisorption of carbon dioxide in a regenerative cartridge is formalized. The authors developed a mathematical model suitable for carrying out imitation studies of the static and dynamic characteristics of the chemisorption process. The model describes mass-exchange, thermal and hydromechanical processes in the gas and solid phases of the regenerative cartridge on chemically bound oxygen. The system of equations of the mathematical model is supplemented by the communication equations, as well as the initial and boundary conditions. The authors proposed an adaptive algorithm for solving equations of a mathematical model that assumes the use of the method of lines in which the template of a finite-difference scheme is chosen from a given set and the condition of a minimum of the integral criterion of accuracy.
\end{abstract}

Keywords-regenerative cartridge; mathematical model; adaptive algorithm; sorption process

\section{INTRODUCTION}

The challenge of protecting individuals or groups of people from exposure to external aggressive environment arose relatively recently. With the development of industry, science and technology, the need for solving this problem has reached the level of economic and political significance. Therefore, research in the field of human protection is quite relevant [1].

One of the main classes of human technical protection systems is life support systems for physiological standards of respiration of staff working in confined spaces or with the use of personal protective equipment.
Most of these systems are based on the principle of selective chemisorption adsorption of carbon dioxide from the exhaled gas mixture [2].

Chemisorption is the process of absorbing substances from the environment by the surface of a liquid or solid body with the formation of chemical compounds.

Advantages of such technical systems of human protection are low energy consumption, mobility, quick access to a preset regime, the ability to manage the composition of separation products, which allows the use of such facilities in various areas of human activity.

A significant number of organizations around the world are engaged in the development and implementation of human protection systems using chemisorption process. As a rule, physical modelling is the main tool, which is costly in terms of resources.

The development of information technology and the growth of computing capacities have made it possible to use the methods of mathematical modelling in solving a wide range of applications at various stages of the life cycle of human protection systems.

Availability of affordable computing capacities opens up new possibilities for research into high-performance algorithms to solve the equations of mathematical models that most accurately describe the character of mass transfer, thermal, hydrodynamic and chemical processes in the air recovery system.

Currently, there are a lot of studies devoted to the development of highly adaptive algorithms for solving equations of mathematical models on computers. 
Mathematical models for regenerative cartridges with chemically bonded oxygen are no exception.

The aim of this paper is to develop an adaptive algorithm for solving the equations of mathematical models with selfadjusting parameters of a calculation scheme from the condition of minimization of the integral criterion.

\section{MAthematical Modeling AND DEVElopMent OF ADAPTIVE ALGORITHM FOR CALCULATION}

The process of chemisorption in the regenerative cartridge is represented as an object, which at the input receives the flux characterized by the vector of input variables $\mathbf{u}(\tau)$, which can include the composition and temperature of the material flow, pressure, velocity, viscosity, etc. In object, input variables $\mathbf{u}(\tau)$ go through the physical and chemical transformations into the variables $\mathbf{y}(\tau)$ or, in other words, the vector $\mathbf{u}(\tau)$ under the action of some process operator $\mathrm{T}$ is converted into a vector of output variables $\mathbf{y}(\tau)$ :

$$
\mathbf{y}(\tau)=\mathrm{T}(\mathbf{u}(\tau))
$$

In the general case, the operator reflects the totality of the simplest physical and chemical transformations occurring in the elements of the object, and has a mixed deterministicstochastic nature [3].

The actual transformations (1) can be described by mathematical model $\mathrm{M}$, the equations of which can be solved using $\mathrm{N}$ algorithms

$$
\widetilde{\mathbf{y}}_{i}(\tau)=\mathrm{M}\left(\mathrm{A}_{i}, \mathbf{u}(\tau)\right), i=\overline{1, \mathrm{~N}}
$$

in the form of a functional operator of input variables $\{u\}$ into the estimation space of output variables $\left\{y_{i}\right\}$. Model (2) is an idealization of transformation (1) and reflects the degree of knowledge about the process. The vector of correct input variables $\mathbf{y}$ does not match the output of models $\widetilde{\mathbf{y}}_{i}$ given that operator $\mathrm{M}$ is an approximate characteristic of $\mathrm{T}$ and is determined by the features of calculation algorithm $\mathrm{A}_{i}$. An essential feature of the identity of operators $T$ and $M$ is the proximity of functions $\widetilde{\mathbf{y}}_{i}$ and $\mathbf{y}(\tau)$ from the viewpoint of norm $\mathbf{R}\left(\mathbf{y}(\tau), \widetilde{\mathbf{y}}_{i}(\tau)\right)[3]$.

In the explicit form, the operator performing the transformation (2) is a closed system of equations and different ratios of empirical character, supplemented with the necessary initial and boundary conditions.

The choice of algorithm $\mathrm{A}_{i}$ is the process of adapting the calculation scheme.

Thus, the solution of the problem of constructing an adaptive algorithm for calculating the equations of the mathematical model with self-adjusting parameters of the calculation scheme from the condition of minimizing the integral criterion is to form a plurality of algorithms for calculating and selecting algorithm $A_{i}$ that provides the proximity of functions $\widetilde{\mathbf{y}}_{i}$ and $\mathbf{y}(\tau)$ from the viewpoint of particular norm $\mathbf{R}\left(\mathbf{y}(\tau), \widetilde{\mathbf{y}}_{i}(\tau)\right)$.

The generalized scheme of the sorption process in the regenerative cartridge, shown in Fig. 1, is considered. The sorption layer is presented in the form of a cylinder of length $L$ and flux section area $S$. The input of the sorption layer $(x=0)$ receives the gas mixture at a rate $G$ and pressure $P_{\text {in }}$, temperature $T_{\text {in }}$ and composition $\mathbf{c}_{\text {in }}=\left\{\mathrm{c}_{1 \text { in }}, \mathrm{c}_{2 \text { in }}, \ldots, \mathrm{c}_{3 \text { in }}\right\}$.

As the gas mixture passes through the granular layer, the selective sorbent adsorption of carbon dioxide and release of oxygen occur. At the output of the sorption layer, the gas mixture is obtained at a rate of $G$, pressure $P_{\text {out }}$, temperature $T_{\text {out }}$ and composition $\mathbf{c}_{\text {out }}=\left\{\mathrm{c}_{1 \text { out }}, \mathrm{c}_{2 \text { out }}, \ldots, \mathrm{c}_{3 \text { out }}\right\}$.

In the mathematical description of the processes in regenerative cartridge, the coordinate axis is arranged along the path of the gas mixture as shown in Fig. 1. The following assumptions are accepted:

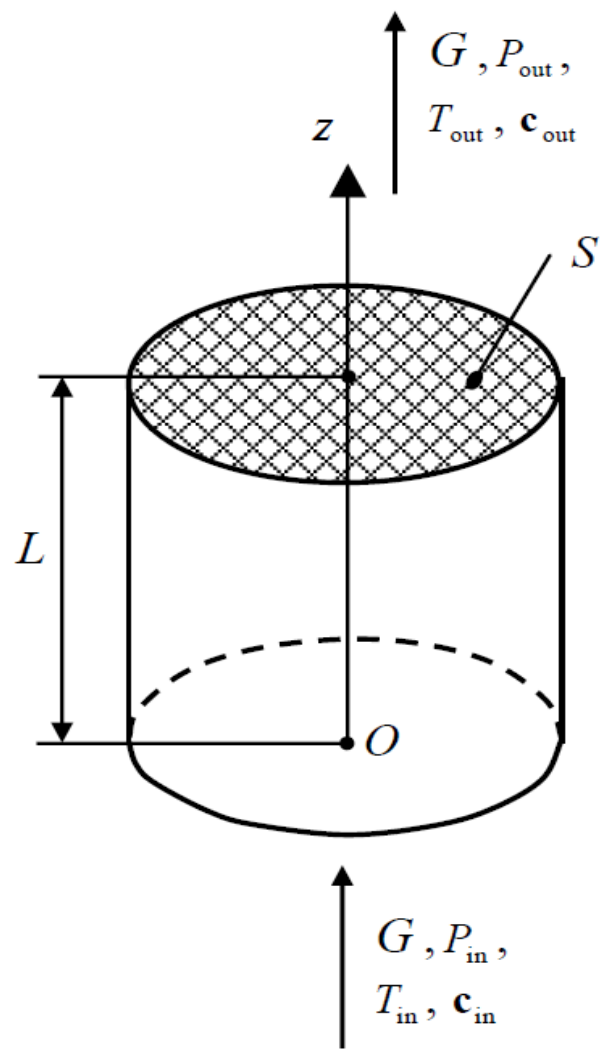

Fig. 1. The scheme of the sorption process in the regenerative cartridge

- Gas mixture is an ideal gas.

- Thermophysical properties of the sorbent granules are constant in time.

- Thermal effect of sorption is constant.

- Heat capacity of gas mixture does not depend on temperature, but it depends on the composition. 
- A velocity of gas mixture is constant along the length of the granular sorbent bed.

- There are no thermal losses into the environment.

- Gas mixture consists of $\mathrm{n}$ components (1 - carbon dioxide, 2 - oxygen, 3 - nitrogen).

According to the accepted assumptions, the mathematical model takes the form of $[4-7]$.

The equations of component-wise material balance are:

$$
\begin{aligned}
& \varepsilon \frac{\partial c_{i}}{\partial \tau}+v \frac{\partial c_{i}}{\partial z}+ \\
& +\rho_{\mathbf{a}} \frac{\partial a_{i}}{\partial \tau}=D_{z, i} \frac{\partial^{2} c_{i}}{\partial z^{2}}, i=\overline{1, n}
\end{aligned}
$$

where $\varepsilon$ is porosity of the adsorbent layer; $c_{i}$ is concentration of the $i$-th component of gas mixture, $\mathrm{mol} / \mathrm{m}^{3} ; t$ is time, $\mathrm{s} ; v$ is velocity of gas mixture along axis $O z, \mathrm{~m} / \mathrm{s} ; z$ is vertical coordinate, $\mathrm{m} ; \rho_{\mathrm{a}}$ is bulk density of adsorbent granules, $\mathrm{kg} / \mathrm{m}^{3}$; $a_{i}$ is the value of sorption of the $i$-th component, $\mathrm{mol} / \mathrm{kg} ; D_{z, i}$ is diffusion coefficient of the $i$-th component along axis $O z$, $\mathrm{m}^{2} / \mathrm{s} ; n$ is the number of gas mixture components.

Trepnel's kinetic equations are:

$$
\frac{\partial a_{i}}{\partial \tau}=\beta_{i} \frac{c_{i}}{\rho_{\mathrm{a}}}\left(1-\frac{a_{i}}{a_{i 0}}\right)^{2}, i=\overline{1, n},
$$

where $\beta_{i}$ is kinetic coefficients for the $i$-th component, $1 / \mathrm{s} ; a_{i 0}$ is ultimate capacity the sorbent for the $i$-th component, $\mathrm{mol} / \mathrm{kg}$.

The equation of thermal balance for sorbent granules is:

$$
\begin{aligned}
& \rho_{\mathrm{a}} \gamma_{\mathrm{a}} \frac{\partial T_{\mathrm{a}}}{\partial \tau}=-\rho_{\mathrm{a}} \sum_{i=1}^{n} \Delta H_{i} \frac{\partial a_{i}}{\partial \tau}+ \\
& +\lambda_{\mathrm{a}} \frac{\partial^{2} T_{\mathrm{a}}}{\partial z^{2}}+K f\left(T-T_{\mathrm{a}}\right),
\end{aligned}
$$

where $\gamma_{\mathrm{a}}$ is specific heat capacity of the sorbent, $\mathrm{J} /(\mathrm{kg} \cdot \mathrm{K}) ; \Delta H_{i}$ is thermal effect of sorption for the $i$-th component, $\mathrm{J} / \mathrm{mol} ; \lambda_{\mathrm{a}}$ is sorbent thermal conductivity coefficient, $\mathrm{W} /(\mathrm{m} \cdot \mathrm{K}) ; K$ is heat transfer coefficient from the granular layer to the gas mixture, $\mathrm{W} /\left(\mathrm{m}^{2} \cdot \mathrm{K}\right) ; f$ is specific surface area of sorbent particles, $\mathrm{m}^{2} / \mathrm{m}^{3} ; T$ is gas mixture temperature, $\mathrm{K} ; T_{\mathrm{a}}$ is sorbent granules temperature, $\mathrm{K}$.

The equation of thermal balance for gas mixture is:

$$
\begin{aligned}
& \varepsilon \rho \gamma \frac{\partial T}{\partial \tau}=-\gamma \nu \rho \frac{\partial T}{\partial z}-P \frac{\partial v}{\partial z}+ \\
& +\varepsilon \lambda \frac{\partial^{2} T}{\partial z^{2}}-K f\left(T-T_{\mathbf{a}}\right)
\end{aligned}
$$

where $\gamma$ is specific heat capacity of gas mixture, $\mathrm{J} /(\mathrm{mol} \cdot \mathrm{K}) ; T$ is gas mixture temperature, $\mathrm{K} ; P$ is gas mixture pressure, $\mathrm{Pa} ; \lambda$ is thermal conductivity coefficient of gas mixture, $\mathrm{W} /(\mathrm{m} \cdot \mathrm{K})$. is:

The Kozeny-Carman equation of momentum conservation

$$
v=-\frac{d^{2} \varepsilon^{3}}{150(1-\varepsilon)^{2} \mu} \frac{\partial P}{\partial \mathrm{z}},
$$

where $d$ is diameter of granules of sorbent particles, $\mathrm{m} ; \mu$ is coefficient of dynamic viscosity of gas mixture, $\mathrm{Pa} \cdot \mathrm{s}$.

The equation of the total mass balance of gas phase is:

$$
\rho=\sum_{i=1}^{n} c_{i}
$$

The equation of state of ideal gas is:

$$
P=\rho R T
$$

The equations of concentration constraints are:

$$
y_{i}=\frac{c_{i}}{\rho}, i=\overline{1, n},
$$

where $y_{i}$ is relative concentration of the $i$-th component of gas mixture, $\mathrm{mol} / \mathrm{mol}$.

The models of physical properties of gas mixture is:

$$
M=\sum_{i=1}^{n} M_{i} y_{i}
$$

where $M$ is molar mass of gas mixture, $M_{i}$ is molar mass of the $i$-th component of gas mixture, $\mathrm{kg} / \mathrm{mol}$.

Thermal conductivity coefficient of gas mixture $\lambda$ (the Brokaw method) [8] is:

$$
\begin{gathered}
\lambda=k \sum_{i=1}^{n} \lambda_{i} y_{i}+\frac{1-k}{\sum_{i=1}^{n} \frac{y_{i}}{\lambda_{i}}}, \\
\lambda_{i}=\lambda_{i, 0}+\Delta \lambda_{i, 1}(T-273.15), i=\overline{1, n},
\end{gathered}
$$


where $k$ is experimental coefficient selected based on the specifics of gas mixture; $\lambda_{i}$ is thermal conductivity coefficient of the $i$-th component of gas mixture, $\mathrm{W} /(\mathrm{m} \cdot \mathrm{K}) ; \lambda_{i, 0}$ is thermal conductivity coefficient of the $i$-th component of gas mixture at $0{ }^{\circ} \mathrm{C}, \mathrm{W} /(\mathrm{m} \cdot \mathrm{K}) ; \Delta \lambda_{i, 1}$ is variation of the thermal conductivity of the $i$-th component of gas mixture at the temperature change of $1{ }^{\circ} \mathrm{C}, \mathrm{W} /\left(\mathrm{m} \cdot \mathrm{K}^{2}\right)$.

The specific heat capacity of gas mixture is:

$$
\gamma=\sum_{i=1}^{n} \gamma_{i} \boldsymbol{y}_{i}
$$

where $\gamma_{i}$ is specific heat capacity of the $i$-th component of gas mixture, $\mathrm{J} /(\mathrm{kg} \cdot \mathrm{K})$.

The dynamic viscosity coefficient of gas mixture calculates by Herning and Zipperer' method $[8,9]$ is:

$$
\begin{gathered}
\mu=\sum_{i=1}^{n} \frac{\mu_{i} y_{i}}{\sum_{j=1}^{n} y_{i} \sqrt{\frac{M_{i}}{M_{j}}}}, \\
\mu_{i}=\mu_{i, 0}+\Delta \mu_{i, 1}(T-273.15), i=\overline{1, n},
\end{gathered}
$$

where $\mu_{i}$ is dynamic viscosity coefficient of the $i$-th component of gas mixture, $\mathrm{Pa} \cdot \mathrm{s} ; \mu_{i, 0}$ is dynamic viscosity coefficient of the $i$-th component of gas mixture at $0{ }^{\circ} \mathrm{C}, \mathrm{Pa} \cdot \mathrm{s}$; $\Delta \mu_{i, 1}$ is variation of dynamic viscosity coefficient of the $i$-th component of gas mixture at temperature change of $1{ }^{\circ} \mathrm{C},(\mathrm{Pa}$. s)/K.

The initial conditions are:

$$
\begin{gathered}
T(z, 0)=T_{0}(z), T_{\mathrm{a}}(z, 0)=T_{\mathrm{a} 0}(z), \\
c_{l}(z, 0)=c_{i 0}(z), a_{i}(z, 0)=a_{i 0}(z), i=\overline{1, n},
\end{gathered}
$$

where $T_{0}(z), T_{\mathrm{a} 0}(z), c_{i 0}(z)$ and $a_{i 0}(z)$ are distributions of model variables in the coordinate at the initial time.

The boundary conditions at the input $(z=0)$ are:

$$
\begin{gathered}
D_{z, i} \frac{\partial c_{i}(0, \tau)}{\partial z}=\frac{G}{S}\left(c_{i}(0, \tau)-c_{i, \text { in }}(\tau)\right), \\
T(0, \tau)=T_{\text {in }}(\tau), \\
\frac{\partial T_{\mathrm{a}}(0, \tau)}{\partial z}=\lambda\left(T_{\mathrm{a}}(0, \tau)-T_{\mathrm{in}}(\tau)\right) .
\end{gathered}
$$

The boundary conditions at the input $(z=L)$ are:

$$
\begin{gathered}
\frac{\partial c_{i}(L, \tau)}{\partial \tau}=0, \frac{\partial T(L, \tau)}{\partial z}=0, \\
\frac{\partial T_{a}(L, \tau)}{\partial z}=0 .
\end{gathered}
$$

Thus, equations (3) - (17) are a mathematical description of mass transfer, thermal and hydrodynamic processes in the regenerative cartridge with chemically bonded oxygen.

To calculate the equations of mathematical model of the regenerative cartridge, the method of lines is used [10].

The system of equations was sampled with respect to variable $z$, thereby turning it into a system of ordinary differential and algebraic equations, for the integration of which the Runge-Kutta method is used with automatic step selection.

The main advantage of the method of lines is that the spatial sampling can be performed with a high density of nodes, and that there is an integration error checking mechanism, and thus, a high accuracy of approximation of solutions both in time and in coordinate $z$ can be achieved.

In accordance with the method of lines, in mathematical model equations (3) - (17), the derivatives are approximated by $z$ finite differences:

$$
\frac{\partial F_{i}(z, \tau)}{\partial z}=\frac{k_{1} F_{i+2}+k_{2} F_{i+1}+k_{3} F_{i}+k_{4} F_{i-1}+k_{5} F_{i-2}+k_{6} F_{i-3}}{k_{0} h_{z}},
$$

$$
\frac{\partial^{2} F_{i}(z, \tau)}{\partial z^{2}}=\frac{F_{i+1}-2 F_{i}+F_{i-1}}{h_{z}^{2}}
$$

where $F_{i}(z, \tau)$ is the value of model variable in the $i$-th node of spatial sampling; $k_{i}$ is weighting factors of difference scheme, $i=\overline{0,6} ; h_{z}$ is step sampling along axis $O z$. 


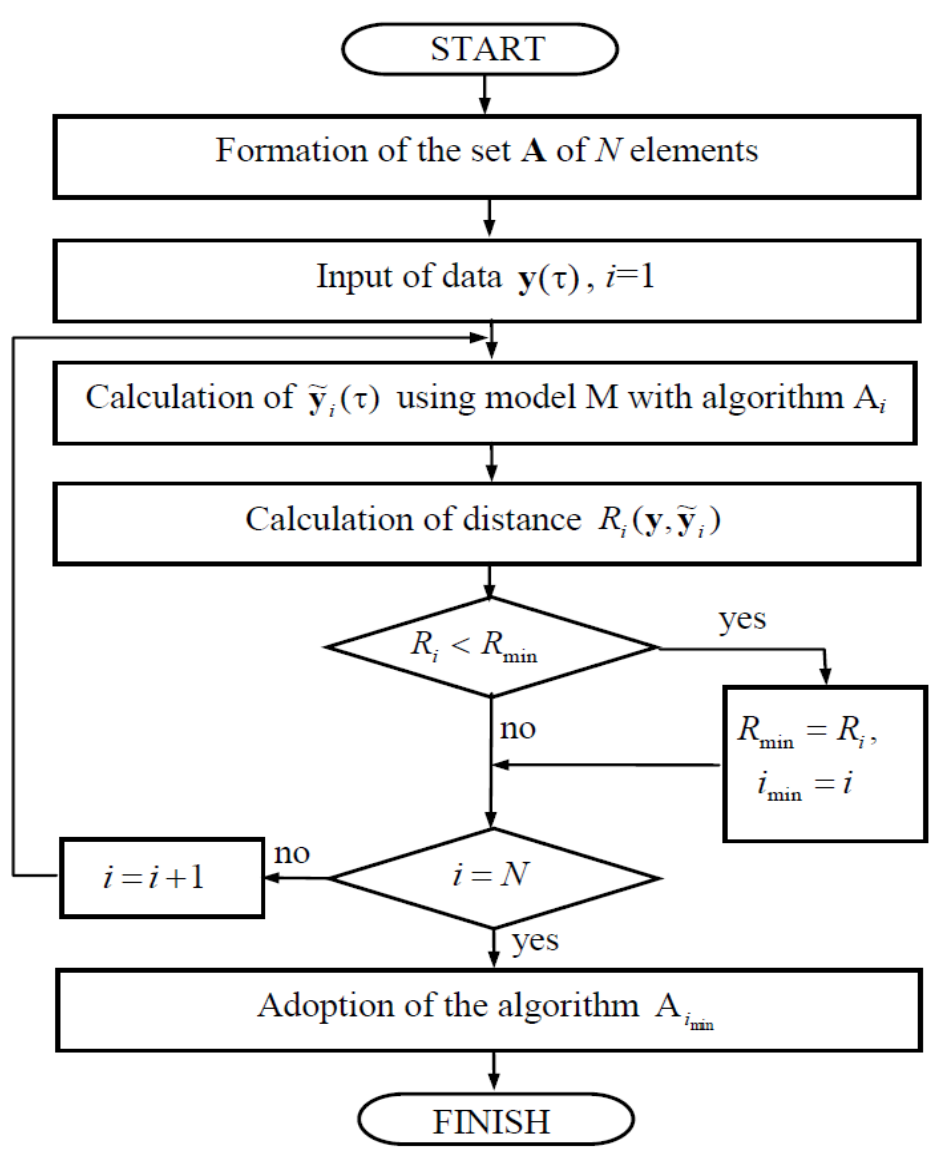

Fig. 2. A block diagram of the adaptation process of the calculation scheme

Fig. 2 is a block diagram of the calculation scheme adaptation of mathematical model equations for the processes in the regenerative cartridge.

The algorithm begins with a process of forming a set from $N$ algorithms. The general view of the approximation of a derivative of the 1st order is represented by the equation (18).

Various $N$ sets of values of weighting coefficients $k_{i}$ allow generating a set of $N$ algorithms. Table I shows the possible sets of weighting coefficients for $N=9$ algorithms [11].

The next step of the algorithm introduced the initial pilot data. Measurement data of output curves $c_{i}(L, \tau)$ are most commonly used as experimental data of chemisorption cartridges.

Further evaluation of $\mathrm{A}_{i}$ algorithm is calculation of the quality score of $R_{i}$ of the form:

$$
R_{i}=\int_{0}^{\tau_{v}}\left(\mathrm{y}(\tau)-\widetilde{\mathbf{y}}_{i}(\tau)\right)^{2} d \tau
$$

where $\tau_{k}$ is the end time of the process of observation, s.
TABLE I. POSSIBLE OPTIONS FOR APPROXIMATION OF THE FIRST DERIVATIVE

\begin{tabular}{|c|c|c|c|c|c|c|c|}
\hline No & $\boldsymbol{k}_{\mathbf{0}}$ & $\boldsymbol{k}_{\mathbf{1}}$ & $\boldsymbol{k}_{\mathbf{2}}$ & $\boldsymbol{k}_{\mathbf{3}}$ & $\boldsymbol{k}_{\mathbf{4}}$ & $\boldsymbol{k}_{\mathbf{5}}$ & $\boldsymbol{k}_{\mathbf{6}}$ \\
\hline 1 & 1 & 0 & 0 & 1 & -1 & 0 & 0 \\
\hline 2 & 2 & 0 & 0 & 3 & -4 & 1 & 0 \\
\hline 3 & 2 & 0 & 1 & 0 & -1 & 0 & 0 \\
\hline 4 & 12 & -1 & 8 & 0 & -8 & 1 & 0 \\
\hline 5 & 6 & 0 & 2 & 3 & -6 & 1 & 0 \\
\hline 6 & 8 & 0 & 3 & 3 & -7 & 1 & 0 \\
\hline 7 & 12 & 0 & 3 & 7 & -11 & 1 & 0 \\
\hline 8 & 12 & 0 & 3 & 10 & -18 & 6 & -1 \\
\hline 9 & 1 & 0 & 0.25 & 0.75 & -1.25 & 0.25 & 0 \\
\hline
\end{tabular}

The next step is a comparison of the value of $R_{i}$ criterion with minimum value $R_{\text {min }}$, which was recorded in the previous steps or set in advance. 
If $R_{i}$ is less than $R_{\min }$, the value of $R_{i}$ and the number of the corresponding algorithm $i_{\min }$ are stored as a new value of the criterion.

In the following step, the current index $i$ is checked. If its value is equal to the number of $N$ algorithms, the enumeration process ends and $\mathrm{A}_{i \mathrm{~min}}$ is adopted as the algorithm, otherwise, the following algorithm $\mathrm{A}_{i+1}$ is processed.

\section{RESULTS OF CALCULATIONS}

Fig. 3 shows the results of calculation of the carbon dioxide output curves for a variety of approximations of the first derivative, where a triangle marks the experimental values of the output concentration of carbon dioxide in the regenerative cartridge.

The number of the algorithm is specified in the curve as shown in Table 1.

Thus, the minimum value of the integral (20) was obtained for difference scheme 8 and amounted to $R_{\min }=R_{8}=57.78$.

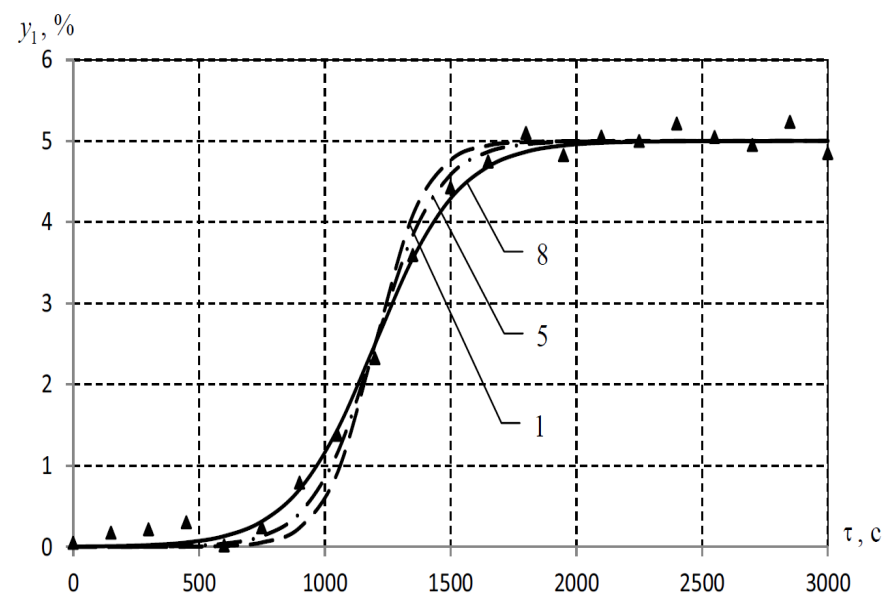

Fig. 3. The results of the calculation of output curves for carbon dioxide

\section{CONCLUSION}

As expected, in the case of the sorption front blurring, the most accurate difference schemes are those that use the largest number of sampling nodes.

However, for extreme sorption fronts, the most appropriate schemes are those with the least amount of sampling points. In the case of the oscillation curves, it is necessary to supplement the set of algorithms with flux limited difference schemes [12].

Thus, the proposed approach is an adaptive algorithm for solving the equations of the mathematical model with selfadjusting parameters of the calculation scheme from the condition of the minimization integral criterion.

\section{Acknowledgment}

This article was supported by the Russian Science Foundation (Agreement No. 15-19-10028).

\section{References}

[1] S.B. Putin, Mathematical Modeling and Control of Air Regeneration Process, Moscow: Mashinostroenie, 2008.

[2] Ju.I. Shumjackij, Industrial Adsorption Processes, Moscow: Koloss, 2009.

[3] I.N. Dorohov, V.V. Men'shikov, System Analysis of the Processes of Chemical Technology. Intellectual Systems and Engineering Creativity in Tasks of Intensification of Chemical and Technological Processes and Production. Moscow: Nauka, 2005.

[4] H. Khajuria, Model-Based Design, Operation and Control of Pressure Swing Adsorption Systems. London: Imperial College London, 2011. https://workspace.imperial.ac.uk/centreforprocesssystemsengineering/P ublic/PhD\%20Thesis/2011\%20PhD\%20Thesis/Khajuria-H-2011-PhDThesis.pdf

[5] A. Agarwal, Advanced Strategies for Optimal Design and Operation of Pressure Swing Adsorption Processes. Delhi: Indian Institute of Technology Delhi, 2010. http://dynopt.cheme.cmu.edu/content/thesis/anshul_thesis.pdf

[6] M. Yavary, H.A. Ebrahim, C. Falamaki, "The effect of number of pressure equalization steps on the performance of pressure swing adsorption process", Chem. Eng. Process.: Process Intensif., vol. 87, pp. 35-44, 2015.

https://doi.org/10.1016/j.cep.2014.11.003

[7] W. Dang, D. Friedrich, S. Brandani, "Characterisation of an automated dual piston pressure swing adsorption (DP-PSA) system", Energy Procedia, vol. 37, pp. 57-64, 2013. https://doi.org/10.1016/j.egypro.2013.05.085

[8] R. Reid, The Properties of Gases and Liquids. New York: McGraw-Hill, 1987.

[9] S.V. Bogoslovskij, The Physical Properties of Gases and Liquids. SpbGUAP: St. Petersburg, 2001. http://window.edu.ru/resource/664/44664/files/2001-0096-0-01.pdf

[10] E. Hairer, G. Wanner, Solving Ordinary Differential Equations II. Stiff and Differential-Algebraic Problems. Springer: Berlin, 1996. http://www.springer.com/us/book/9783540604525

[11] G.A. Korn, T.M. Korn, Mathematical Handbook for Scientists and Engineers: Definitions, Theorems, and Formulas for Reference and Review. New York: McGraw-Hill Book Company, 1968.

[12] A. Harten, S. Osher, "Uniformly high-order accurate nonoscillatory schemes. I", SIAM J. Numer. Anal., vol. 24, no. 2, pp. 279-309, 1987. https://doi.org/10.1137/0724022 